\title{
POLITIQUE PUBLIQUE ET MANAGEMENT PUBLIC, DE NOUVELLES FRONTIERES ?
}

Cécile Desmarais, David Giauque

AIRMAP | «Gestion et management public »

2016/2 Volume $4 / n^{\circ} 4$ | pages 1 à 7

Article disponible en ligne à l'adresse :

http://www.cairn.info/revue-gestion-et-management-public-2016-2-page-1.htm

\section{Pour citer cet article :}

Cécile Desmarais, David Giauque, « Politique publique et management public, de nouvelles frontières ? », Gestion et management public 2016/2 (Volume 4 / n 4), p. 1-7.

Distribution électronique Cairn.info pour AIRMAP .

(C) AIRMAP . Tous droits réservés pour tous pays.

La reproduction ou représentation de cet article, notamment par photocopie, n'est autorisée que dans les limites des conditions générales d'utilisation du site ou, le cas échéant, des conditions générales de la licence souscrite par votre établissement. Toute autre reproduction ou représentation, en tout ou partie, sous quelque forme et de quelque manière que ce soit, est interdite sauf accord préalable et écrit de l'éditeur, en dehors des cas prévus par la législation en vigueur en France. Il est précisé que son stockage dans une base de données est également interdit. 


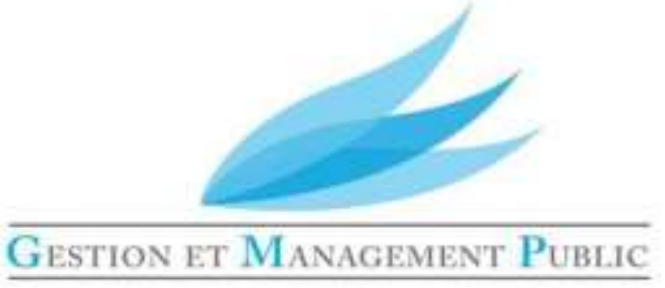

\section{POLITIQUE PUBLIQUE ET MANAGEMENT PUBLIC, DE NOUVELLES FRONTIÈRES?}

\section{Cécile DESMARAIS ${ }^{1}$, David GIAUQUE ${ }^{2}$}

\section{Introduction: l'interface politico- administrative sous la loupe}

La publicitude d'une activité (c'est-à-dire son caractère public), se traduit par l'importance prise par la dimension politique (par rapport à la dimension économique) comme principe de fonctionnement de cette activité (Bozeman, 2007). Ainsi, le fonctionnement des organisations publiques est-il foncièrement traversé par des recouvrements et des empiètements entre les dimensions politiques et administratives (Peters, Pierre, 2004). La frontière, bien souvent mouvante, entre politiques publiques et management public est au cœur du fonctionnement des organisations publiques. Du reste, la politisation de l'administration (Rouban, 2005), tout comme la managérialisation progressive du politique (Gaulejac de, 2005), constituent un indice de l'imbrication de ces deux sphères et surtout des dérives possibles au cœur des univers bureaucratiques et politiques.

Les sciences politiques se sont intéressées depuis un certain temps à cette problématique des relations politicoadministratives. II existe donc une tradition de recherches sur cette question, bien que celleci soit naturellement plus encline à aborder des thématiques plus institutionnelles et

\footnotetext{
1 HEIG-VD, HES-SO, Institut interdisciplinaire du développement de l'entreprise (IIDE)

2 Université de Lausanne, Institut d'études politiques, historiques et internationales (IEPHI)
}

macros, délaissant, de ce fait, une analyse centrée plus particulièrement sur les acteurs. Des travaux plus récents, de sociologie politique notamment, ont entrepris de s'intéresser à la composition des arènes politiques et administratives, autrement dit aux élites politiques et administratives (Emery et al., 2014; Mach et al., 2011). Par contre, il faudra attendre un peu plus longtemps avant de voir apparaître des recherches au sein de la discipline du management public sur cet objet. Ainsi, la domination traditionnelle des sciences politiques sur l'analyse des interactions entre sphère politique et sphère administrative s'estompe tandis que d'autres disciplines davantage centrées sur des problématiques de management public s'immiscent dans les sujets traditionnellement politiques (Gibert, 2002).

Par ailleurs, les équilibres traditionnels entre sphère politique et sphère administrative sont réinterrogés par la diffusion des principes du nouveau management public. Ceux-ci rompent avec la conception wébérienne qui envisageait la séparation entre politique et administration à travers la soustraction des hauts fonctionnaires à l'arbitraire politique. Ils diffusent une vision normative alternative fondée sur une redéfinition des sphères d'action du politique et de l'administration et une dépendance accrue des fonctionnaires vis-à-vis du politique. L'intérêt porte dès lors bien souvent sur la question des compromis, ou négociation (bargain en anglais) entre les acteurs politiques et administratifs notamment dans le cadre de leurs difficiles partage des tâches. Car, en ce domaine, il faut bien admettre que les activités administratives 
sont fondamentalement politiques, de même que les actes politiques ont une portée administrative tout aussi évidentes (Elston, 2016; Hood, Lodge, 2006). Par ailleurs, loin d'assister à une homogénéisation de ces compromis politico-administratifs, il faut plutôt noter une mise en œuvre extrêmement différenciée dans les différents pays et même au sein d'une même nation. En matière de relations politico-administratives, les traditions politiques et administratives ont une influence majeure (Bevir et al., 2003; Knill, 1998; Painter, Peters, 2010). Ces interactions entre politique et administration sont par ailleurs rendues difficiles par la complexité élevée des relations existant entre ces deux sphères. Les réformes entreprises ces trente dernières années visant à rendre l'administration plus efficiente, et parfois plus efficace (encore que les deux n'aillent pas forcément de pair comme l'a démontré la littérature scientifique contemporaine), de même que le regain de populisme politique de ces dernières années ont contribué à rendre l'interface entre politique et administration particulièrement délicate (Giauque, Emery, 2016). Aussi, il n'est pas rare d'entendre les politiciens faire campagne en critiquant vertement le fonctionnement des administrations, celles-là même qu'ils auront la tâche de diriger en cas d'élection. Si la relation politico-administrative nécessite une certaine confiance réciproque entre acteurs, les dénonciations politiques populistes des dysfonctionnements administratifs n'y contribuent guère (Audette-Chapdelaine, 2016; Luhmann, 1979). Autrement dit, si la coopération est souhaitable et si les activités politiques et administratives sont interdépendantes, elles sont aussi bien souvent en concurrence notamment en ce qui a trait à leur logique de fonctionnement et aux valeurs sur lesquelles elles se fondent.

L'analyse de ces relations politicoadministratives, de cette interface, est d'autant plus importante que nous assistons d'une part à une "dé-liaison" entre des disciplines qui, hier encore, se parlaient et qui ne le font plus ou trop rarement. "Le constat que nous faisons est le suivant: les investigations sur les processus de décision publique, les recherches sur l'administration et les réflexions sur l'Etat ne relèvent plus, aujourd'hui mais depuis longtemps, des mêmes sous-disciplines ni ne font l'objet des mêmes travaux" (Bezes, Pierru, 2009, p. 1). De façon assez légitime, l'intérêt des spécialistes des politiques publiques s'est porté principalement sur les rôles des acteurs politiques, des groupes d'intérêt dans les étapes d'une politique publique (mise à l'agenda; programmation; mise en œuvre; évaluation). De plus, ces différentes étapes ont fait l'objet de nombreuses études, les acteurs de l'administration publique n'apparaissant la plupart du temps que dans celle portant sur la mise en œuvre des politiques publiques. D'autre part, I'hyperspécialisation académique a conduit à la création et à l'autonomisation de la discipline "administration publique», contribuant à rompre un dialogue fécond avec des disciplines des sciences sociales et politiques qui traditionnellement portent un intérêt pour les acteurs et leurs interactions, pensons notamment à la sociologie des organisations, à l'anthropologie, à l'ethnologie et à la politique comparée, pour n'en mentionner que quelques-unes. Au niveau académique, les cursus scientifiques portant spécifiquement sur l'administration publique sont nés, la plupart du temps, dans des facultés de droit, voire de sciences économiques, plus récemment dans les facultés des sciences humaines et sociales. En conséquence, les réflexions scientifiques contemporaines relatives à l'administration publique sont portées par des considérations juridiques, économiques et managériales qui ne favorisent pas la prise en compte des acteurs et de leurs interactions. Et ce malgré le fait que l'après seconde guerre mondiale est une période féconde en termes de réflexions sociologiques sur la bureaucratie, en Europe et aux Etats-Unis (Blau, 1955; Crozier, 1963; Gouldner, 1968; Merton, 1940; Selznick, 1966; 1957). 


\section{Contenu du dossier: différentes approches de la frontière politico- administrative}

C'est pourquoi, au-delà de l'analyse des équilibres et déséquilibres politicoadministratifs, ainsi que de leur signification, il est important d'observer le fonctionnement détaillé des interactions existant entre la sphère politique et la sphère administrative : comment les enjeux mutuels de chacune de ces sphères se nourrissent-ils mutuellement? Quels sont les processus d'influence réciproques entre légitimité économique, légitimité administrative et légitimité politique? En quoi les évolutions du management public et l'influence du nouveau management public affectent-ils la frontière politico-administrative et les relations entre leurs acteurs ?

Ces questions ont fait l'objet d'une section thématique "Politique Publique et management public " au sein du colloque COSPOF 2015, sixième congrès des associations francophones de science politique, qui s'est tenu du 5 au 7 février 2015 à l'Université de Lausanne. Les articles de ce numéro spécial, issus des 13 communications sélectionnées en vue d'une présentation dans le cadre du colloque, ont ensuite été soumis au processus d'évaluation de la revue Gestion et Management Public.

Ceux-ci abordent la question des interactions entre politique publique et management public à travers le double mouvement de managérialisation du politique et de politisation du management. L'actualité donne en effet à voir une managérialisation croissante de la sphère politique, en lien avec une relation entre sphère administrative et sphère politique qui est davantage dialectique que subordonnée (de Visscher, 2004). Inversement, la politisation du management semble être une conséquence du nouveau management public, à travers notamment les nominations politiques des hauts cadres de l'administration publique. Mais son analyse doit être menée avec précaution car la politisation peut prendre des formes extrêmement différenciées selon les systèmes institutionnels (Peters, Pierre, 2004), comme nous le mentionnions auparavant.

Les articles de ce dossier confirment l'existence de ce double mouvement qui $s^{\prime}$ inscrit à trois niveaux d'analyse : le niveau de la place des enjeux managériaux dans les considérations politiques, celui de la place de l'expertise et des experts dans les processus managériaux et politiques et enfin celui de la relation entre haute fonction publique et politique.

\subsection{L'imbrication des enjeux managériaux dans la construction de la légitimitié politique}

Ainsi deux articles portent principalement sur l'imbrication des enjeux managériaux dans la construction de la légitimité politique.

L'article de Muriel Michel-Clupot et Serge Rouot, "Une managérialisation du discours politique. L'exemple de travaux parlementaires sur les crises de la finance " s'intéresse à la contamination des travaux parlementaires par l'approche managériale. Ce texte s'attaque à l'épineuse question de la managérialisation de l'Etat et plus spécifiquement du discours politique. II s'appuie pour cela sur l'analyse textuelle de deux rapports récents publiés suite à des enquêtes parlementaires en France (commission d'enquête sur les produits financiers à risque souscrits par les acteurs publics locaux, et mission commune d'information sur le fonctionnement, la méthodologie et la crédibilité des agences de notation).

Les auteurs mobilisent l'approche des parties prenantes pour montrer, à travers l'audition des principaux acteurs concernés (les "victimes » de ces pratiques, les responsables de telles pratiques, ainsi que les experts consultés), comment la logique économique et managériale pénètre le discours politique, notamment à travers la reconnaissance ou 
non de la crise d'une part, et l'expression d'une volonté de réglementation d'autre part. Il apparaît ainsi une porosité entre les discours économique et politique, ainsi qu'une influence évidente des experts consultés, dont les arguments sont repris dans les conclusions des parlementaires.

L'article de Jean-Francois Savard, "Réformes de la politique autochtone au Canada : le jeu de blâme donne-t-il une cohérence au discours ? " décrit les jeux subtils par lesquels le discours politique renforce les approches de gestion et convoque celles-ci comme des arguments supplémentaires. Pour cela, il décortique le discours tenu par le gouvernement canadien au sujet des réformes de la politique autochtone. Mobilisant une approche originale qui combine le "cohérentisme " et le "jeu du blâme ", à travers une mesure de la cohérence appelée " approche relationnelle " appliquée à plus de 300 pages de retranscription des débats à la Chambre des communes sur le sujet traité par l'article, l'auteur parvient à montrer que le gouvernement justifie ses réformes dans un discours relativement cohérent, articulé en 10 arguments centraux, tout en activant une double stratégie du jeu de blâme. Cette stratégie consiste, tout d'abord, à rejeter le blâme issu des partis de l'opposition sur euxmêmes, pour ensuite dévier ce blâme en se positionnant comme l'allié des Autochtones, promoteur de leur économie et de leur développement économique. Au-delà de l'objet d'analyse, l'approche développée par l'auteur ouvre la voie à de nouvelles recherches basées sur la cohérence du discours tenu dans divers champs de politiques publiques. Cet article traite, par ailleurs, d'une problématique très sensible au Canada puisqu'elle concerne les politiques publiques en lien avec les populations autochtones. Si le gouvernement Trudeau actuel semble plus sensible aux problématiques spécifiques auxquelles sont confrontées les populations autochtones, les relations entre le Gouvernement et ces mêmes populations étaient plus conflictuelles sous le gouvernement conservateur de Harper. Ce dernier estimait que les solutions devaient nécessairement passer par un recours plus important au marché et que la stimulation de l'esprit entrepreneurial des populations autochtones leur permettrait de faire face à leurs problèmes (Gutstein, 2014). Cet article traite donc d'une question de très grande actualité au Canada.

\subsection{La place des experts dans l'administration et dans les systèmes de légitimation politique}

La relation entre politiques publiques et administration interroge par ailleurs la place des experts dans l'administration et dans les systèmes de légitimation politique.

L'article de Damien Wirths, "Les clauses d'évaluation sectorielles : outils d'ajustement ou de stratégie politique ? Le cas de l'interdiction de la mendicité à Lausanne ", interroge la place des experts en analysant l'impact des clauses qui imposent d'évaluer les effets d'une loi. Partant du constat selon lequel l'évaluation des politiques est un phénomène en pleine croissance, qui s'institutionnalise dans de nombreux pays, au rang desquels la Suisse figure dans les premières places, il s'interroge sur l'utilisation réelle qui peut être faite de ces dispositifs. En effet, il s'agit de comprendre si l'évaluation favorise des décisions qui sont fondées sur l'efficacité (evidence-based policymaking) ou sur l'acceptabilité (opinion-based policymaking), sachant que la théorie met l'accent sur le fait que le premier concept semble prendre le pas sur le second. Pour cela l'auteur analyse le cas de l'interdiction de la mendicité au sein de la Ville de Lausanne en Suisse, cas qui se révèle crucial en ce qu'il permet de contredire la thèse selon laquelle l'évaluation permettrait nécessairement de construire des politiques sur des résultats plutôt que sur des opinions. Les données analysées sont issues du débat qui s'est déroulé en 2013 au sein du Conseil communal de Lausanne (organe législatif) autour de son règlement de police. La proposition d'y introduire une clause d'évaluation a donné lieu à un débat nourri, mobilisant tous les partis du Conseil, permettant au chercheur d'observer un phénomène précédemment considéré comme inaccessible à l'étude scientifique, à travers un enregistrement des 
débats, et des entretiens semi-directifs avec certains parlementaires. La conclusion de ce travail montre qu'en effet, le recours aux experts est surtout considéré par les parlementaires comme une façon de poursuivre la politique par d'autres moyens, l'avis de l'expert étant instrumentalisé au service d'un dessein politique ou n'étant mobilisé que s'il sert ce dessein, les expertises permettant alors de sortir de nouvelles cartes dans le jeu politique.

\subsection{Ethos des cadres dirigeants publics et brouillage des frontières politico- administratives}

Enfin, le brouillage et la redéfinition des frontières entre politique publique et administration publique a un effet central sur l'éthos des hauts fonctionnaires, la définition de leur rôle et les valeurs qu'ils professent.

L'article de Isabelle Fortier, Yves Emery et Rachel Roldan, " Des conditions et enjeux de la politisation de l'administration publique au regard de l'ethos public: une étude comparée (Canada/Suisse) de l'expérience des cadres dirigeants ", propose une lecture de la dynamique sociale caractérisant l'ethos public sous l'angle de la politisation de l'administration à travers le regard de ceux qui la vivent. II s'appuie sur des récits de vie de cadres dirigeants qui œuvrent dans différents secteurs de l'administration publique, en Suisse et au Canada. Les auteurs analysent les représentations des répondants sur ce qui est possible, normal ou désirable au regard du maintien de l'éthos public ou de sa transformation. Sur la base de ces représentations ils mettent l'accent sur les différences du vécu des acteurs qui s'appuient sur des fonctionnements institutionnels différenciés. L'analyse distingue la politisation formelle (substitution de critères partisans aux critères basés sur le mérite dans la carrière des hauts fonctionnaires), la politisation fonctionnelle (implication des hauts fonctionnaires dans la définition des politiques) et la politisation administrative (présence de conseillers politiques qui interfèrent dans la relation entre politiques et hauts fonctionnaires). II montre que dans les deux pays, les sources de fierté des cadres dirigeants sont rattachées à la conviction de servir l'intérêt général et de contribuer à l'évolution de la société, dans une action conçue à long terme mais que cela s'exprime différemment en termes d'ethos public et produit des réactions différentes. Cet article fait écho aux très nombreuses publications récentes portant sur l'éthos public dans les différents pays de I'OCDE (Farnham, Horton, 2005; Fortier, Emery 2012; John, Johnson, 2008) et interroge en conséquence également les valeurs, les identités ainsi que les motivations des cadres dirigeants publics.

\section{Ouverture}

Les différents articles de ce numéro spécial illustrent le fait que si la légitimité politique est aujourd'hui concurrencée par le principe de performance productive propre à l'univers managérial, le pouvoir politique est toutefois en mesure d'utiliser cette nouvelle forme de légitimité au service de projets politiques. De même, si la politisation de l'administration constitue aussi une réalité, notamment par le truchement de la consultocratie, la sphère administrative conserve une autonomie importante dans la définition et la mise en œuvre des politiques publiques. Aussi, il ne fait guère de doute que les relations politicoadministratives se trouvent actuellement en pleine redéfinition, notamment du fait des réformes étatiques, de l'apparition de nouvelles politiques publiques nécessitant une expertise croissante et des réformes managériales entreprises au sein des administrations publiques elles-mêmes. Ces changements vont, sans aucun doute, influencer et retravailler l'interface politicoadministrative durant ces prochaines décennies. Ceci étant, si, comme l'ont démontré les articles intégrés dans ce numéro spécial, l'interface politico-administrative s'avère complexe et délicate et qu'elle résiste à toute généralisation excessive, elle s'avère primordiale dans le développement et la mise en œuvre des réformes actuellement en cours dans la majorité des pays de l'OCDE. 
Par exemple, il a été démontré dans la littérature contemporaine que la définition et I'utilisation de systèmes d'information en vue de conduire les affaires publiques dépendent très largement de l'implication des acteurs politiques et administratifs et de leur confiance mutuelle (Kroll, 2015). De même, l'efficience des systèmes de mesure de la performance est fonction de l'implication des acteurs des sphères politiques et administratives (Yang, Hsieh, 2007). Ces constats appellent ainsi à la réalisation de nouvelles recherches questionnant les déterminants, les fonctionnements et les " outputs" des relations politicoadministratives. De même, des comparaisons pourraient être utiles afin de mieux comprendre quels sont les effets des institutions politiques, des structures et cultures administratives sur l'interface politico-administrative. Quoi qu'il en soit, la relation entre politique publique et administration publique s'avère d'une très grande complexité et démontre une certaine résistance à la modélisation et à la simplification. Elle s'exprime de manière diverse selon les contextes et les enjeux et les interprétations différenciées dont elle fait l'objet auprès des différentes catégories d'acteurs. Ainsi, le présent dossier contribue, nous l'espérons, à illustrer le fait que le système de relations entre administration et politique mérite d'autres et futures investigations scientifiques.

\section{Articles du varia}

Ce varia comprend trois articles.

Franck Brillet et Isabelle Sauviat dans leur article intitulé "L'avenir incertain des dispositifs d'anticipation $\mathrm{RH}$ au sein des universités françaises: entre recherche de sens et perte de sens " s'intéressent au travers d'une recherche-action à la façon dont la dialectique fin/moyens impacte les pratiques de gestion prévisionnelle des effectifs, des emplois et des compétences dans les universités françaises. Ils montrent, que l'adoption de la GPEEC dans deux universités renvoie à des situations très différentes. Prenant appui sur la théorie du sense making, ils expliquent comment la GPEEC correspond à une démarche de " construction de sens ", soit de " perte de sens ".

Jocelyne lentile-Yalenos, Eric Thivant et Alain Roger dans leur article intitulé « Le Nouveau Management Public conduit-il à un rapprochement public-privé ? Une analyse à partir des procédures d'évaluation du personnel " examinent dans quelle mesure les caractéristiques des systèmes d'évaluation du personnel dans le secteur public répondent à des normes universelles ou sont au contraire spécifiques à celui-ci. Pour mener cette étude ils recourent à l'analyse lexicométrique des documents qui définissent les conditions d'application de ces systèmes dans deux organisations publiques et deux entreprises privées. Les deux organisations du secteur public présentent plusieurs caractéristiques des cultures bureaucratiques, mais elles s'en éloignent en développant la responsabilité et la flexibilité, deux piliers du " Nouveau Management Public ».

Isabelle Horvath et Gaëlle Dechamp dans leur article "Quand les pouvoirs publics favorisent la proximité pour stimuler la créativité du territoire » examinent, dans quelle mesure la proximité des acteurs, serait un levier de production d'idées. Elles montrent que la proximité spatiale n'est pas suffisante pour rapprocher deux activités différentes et que c'est surtout la proximité relationnelle qui provoque la créativité organisationnelle et inter-organisationnelle.

\section{Références}

AUDETTE-CHAPDELAINE M. (2016), "Sensemaking and the political-administrative interface: the challenges of strategically steering and managing a local public service", International Review of Administrative Sciences, 82, 3, 454-471.

BEVIR M., RHODES R.A.W., WELLER P. (2003). "Traditions of governance: interpreting the changing role of the public sector", Public Administration, 81, 1, 1-17.

BEZES P., PIERRU F. (2009), "Les dé-liaisons dangereuses. Les trajectoires croisées de la sociologie de l'Etat, de l'analyse des politiques 
publiques et de la science administrative. Un essai de comparaison franco-américaine", 10ème Congrès de l'Association Française de Science Politique, Grenoble.

BOZEMAN B. (2007), Public Values and Public Interest: Counterbalancing Economic Individualism, Washington, DC: Georgetown University Press.

BLAU P.M. (1955), The Dynamics of Bureaucracy. Chicago, IL: University of Chicago Press.

CROZIER M. (1963), Le phénomène bureaucratique. Paris: Seuil.

ELSTON, T. (2016). Conflict between Explicit and Tacit Public Service Bargains in U.K. Executive Agencies. Governance, n/a-n/a.

EMERY, Y., GIAUQUE, D., REBMANN, F. (2014). The slow transformation of Swiss federal administrative elites. International Review of Administrative Sciences, 80(4), 687-708.

FARNHAM D., HORTON S. (2005), "Origins, development and decline of the public service ethos in the British civil service: an historical institutional analysis", Colloque: the Reforming the public sector: what about the citizens?, Bern.

FORTIER I., EMERY Y. (2012), "L'ethos public en tant que processus social dynamique", Pyramides, revue du CERAP, 22, 2, 83-114.

GAULEJAC DE V. (2005). La société malade de la gestion. Paris: Seuil.

GIAUQUE D., EMERY Y. (2016), L'acteur et la bureaucratie au XXIème siècle, Québec: Les Presses de l'Université Laval.

GOULDNER, A. W. (1968). Patterns of Industrial Bureaucracy. New York: The Free Press.

GUTSTEIN, D. (2014). Harperism. How Stephen Harper and his think tank colleagues have transformed Canada. Toronto: James Lorimer \& Company LTD.

HOOD, C., LODGE, M. (2006). The Politics of Public Service Bargains: Reward, Competency, Loyalty and Blame. Oxford: Oxford University Press.

JOHN P., JOHNSON M. (2008), "Is there still a public service ethos?", in PARK A., CURTICES J., THOMSON K., PHILLIPS M., JOHNSON M., CLERY E. (Eds.), British Social Attitudes: The 24th Report, London: Sage, 105-123.

KNILL, C. (1998), "European Policies: The Impact of National Administrative Traditions", Journal of Public Policy, 18, 1, 1-28.
KROLL A. (2015), Drivers of Performance Information Use: Systematic Literature Review and Directions for Future Research. Public Performance \& Management Review, 38, 3, 459-486.

LUHMANN N. (1979). Trust and Power. Chichester: Wiley.

MACH A., DAVID T., BÜHLMANN F. (2011), La fragilité des liens nationaux. La reconfiguration de l'élite du pouvoir en Suisse, 1980-2010. Actes De La Recherche En Sciences Sociales, 190, 78-107.

MERTON R. (1940), "Bureaucratic Structure and Personality", Social Forces, 18, 560-568.

PETERS B.G., PIERRE J. (Eds.) (2004), The politicization of the civil service in comparative perspective: A quest for control. Routledge.

PAINTER M., PETERS B.G. (Eds.). (2010). Tradition and Public Administration. New York: Palgrave Macmillan.

ROUBAN L. (2005), "Politicization of the Civil Service", in PIERRE J., PETERS B.G. (Eds.), Handbook of Public Administration, London: Sage.

SELZNICK P. (1966), TVA and the Grass Roots. A study in the Sociology of Formal Organization. New York: Harper \& Row.

SELZNICK P. (Ed.) (1957), Leadership in administration: a sociological interpretation. Evanston White Plains: Row \& Peterson.

YANG K., HSIEH J.Y. (2007), "Managerial Effectiveness of Government Performance Measurement: Testing a Middle-Range Model", Public Administration Review, 67, 5, 861-879. 